\title{
Achieving Allocative Efficiency in Healthcare: Nice in Theory, not so NICE in Practice?
}

\author{
Mike Paulden • Christopher McCabe • \\ Jonathan Karnon
}

Published online: 6 March 2014

(c) Springer International Publishing Switzerland 2014

\section{Introduction}

Eckermann and Pekarsky [15] have written a thoughtprovoking paper that challenges a number of existing concepts, including methods for determining the costeffectiveness threshold for appraising new health technologies for potential adoption into a public healthcare system.

Previously proposed approaches to defining the threshold have included the estimation of the societal monetary value of a QALY to inform the net monetary benefits of new technologies, where the technology is funded if the net benefits are positive. Alternative approaches aim to represent opportunity cost, either by focusing on the QALYs forgone from the displacement of services to generate resources to fund new technologies, or as a retrospective estimate of expected QALY gains from budget expansion or contraction at the margin [1-7].

The authors' primary argument is that proposed approaches to defining the threshold have misspecified the opportunity cost of adopting new technologies. In a budgetconstrained healthcare system that is not operating at allocative efficiency (i.e. all real-world public healthcare

\section{Paulden · C. McCabe}

Faculty of Medicine and Dentistry, University of Alberta, Suite 736 University Terrace, 8303112 Street, Edmonton, AB T6G 2T4, Canada

e-mail: paulden@ualberta.ca

C. McCabe

e-mail: mccabel@ualberta.ca

J. Karnon ( $\square)$

School of Population Health, University of Adelaide, Level 7,

178 North Terrace, Adelaide, SA 5005, Australia

e-mail: jonathan.karnon@adelaide.edu.au systems), Eckermann and Pekarsky argue that the threshold should reflect optimal approaches to two decision processes involved in the reallocation of funds: the displacement of services to free up resources, and the subsequent adoption of new technologies or expanded services.

The following section provides a stylised example to illustrate the approach suggested by Eckermann and Pekarsky, followed by a discussion of its relevance to current technology reimbursement processes. In common with Eckermann and Pekarsky, we use the National Institute for Health and Care Excellence (NICE) in the UK as an exemplar. The final section considers the feasibility of adapting current reimbursement processes to one in which the proposed, theoretically derived estimate of the health shadow price (opportunity cost) can be routinely applied.

\subsection{Opportunity Cost and the Optimal Cost- Effectiveness Threshold}

Consider a public healthcare system with a fixed budget. In this health system, suppose that the most cost effective expansion of existing health services is associated with an incremental cost-effectiveness ratio (ICER) of $\$ 2,000$ per QALY, and the least cost-effective health activity has an ICER of $\$ 100,000$ per QALY. A new health technology is under assessment for potential adoption into the health system and is determined to have marginal budget impact and an ICER of $\$ 2,500$ per QALY. To generate the resources required to fund the new technology, existing healthcare services must be displaced. Due to suboptimal disinvestment processes, the least cost effective activity is not displaced, but rather an alternative healthcare activity with an ICER of $\$ 50,000$ per QALY.

Under the conventional framework adopted by NICE in the UK, the cost-effectiveness threshold in this example 
would be set at $\$ 50,000$ per QALY, representing the shadow price of the healthcare budget in contraction. Since the ICER of the new technology falls comfortably below this threshold, the technology would be recommended. The reasoning behind this decision is that for every $\$ 50,000$ reallocated to the new technology, a single QALY would be expected to be lost through the displacement of the least cost effective existing health activity. However, 20 QALYs would be expected to be gained from adopting the new technology (which has an ICER of $\$ 2,500$ per QALY), resulting in an expected net gain in population health of 19 QALYs.

If the objective of the reimbursement authority is simply to ensure that the new technology does not displace more health than it provides, this conventional approach to determining the threshold is appropriate. However, an alternative objective might be to maximise the value of the health produced from the expenditure of the healthcare budget (i.e. move towards allocative efficiency across the whole healthcare system). In this situation, Eckermann and Pekarsky note that the conventional 'displacement threshold' approach is suboptimal in two respects.

Firstly, displacement may not be optimal. If the displaced service has an ICER of $\$ 50,000$ per QALY, but the least cost effective service currently provided has an ICER of $\$ 100,000$ per QALY, then there is inefficiency in displacement that prevents the healthcare system from achieving allocative efficiency. In this example, the loss from inefficiency in displacement is 1 QALY for every $\$ 100,000$ displaced: the displaced service is losing 2 QALYs per $\$ 100,000$ rather than the 1 QALY that would be lost if the least cost effective service was displaced.

Secondly, the use of displaced resources might be viewed as being subject to an adoption decision, rather than a fait accompli that the resources will fund a new technology. In this case, the resources may be used to fund the new technology, or to fund the expansion of the most cost effective existing service. For every $\$ 100,000$ reallocated to the new technology, population health is expected to increase by 40 QALYs, but allocating the $\$ 100,000$ to the most cost effective service in expansion would instead produce 50 QALYs.

In this example, the optimal reallocation process would replace the least cost effective service (ICER of $\$ 100,000$ per QALY) and expand the most cost effective service (ICER of \$2,000 per QALY). The resulting health gain per $\$ 100,000$ reallocated is 49 QALYs (50 gained minus 1 displaced). If the new technology is adopted at the expense of the service with an ICER of $\$ 50,000$, the net health gain is 38 QALYs (40 gained minus 2 displaced). Compared with the optimal process, 11 QALYs are forgone per $\$ 100,000$ of reallocation.

Based on this exposition of the reallocation process, Eckermann and Pekarsky define the health shadow price, and hence the cost-effectiveness threshold that will lead a healthcare system towards allocative efficiency. In this example, the threshold would be:

$$
\left(\frac{1}{2,000}+\frac{1}{50,000}-\frac{1}{100,000}\right)^{-1}=\$ 1,961 \text { per QALY }
$$

To demonstrate, if the price of the new technology is reduced such that the ICER is lowered to $\$ 1,961$ per QALY, and the new technology is adopted at the expense of the service with an ICER of $\$ 50,000$ per QALY, then the net health gain is 49 QALYs (51 gained minus 2 displaced), the same as the optimal reallocation process.

In practice, the least cost effective service in contraction may well be more costly and less effective than its comparator, and the most cost effective service in expansion may be more effective and less costly than its comparator. If either of these scenarios occurs, a new technology would only be funded if it was more effective and less costly than its comparator.

\section{NICE in Practice?}

If an accurate estimation of the health shadow price is available and this is used to inform the cost-effectiveness threshold, then the process of funding new technologies would be expected to achieve the same gain in allocative efficiency of the healthcare system as the best alternative process of displacing the most inefficient service to expand the most cost effective service.

There are, however, a number of concerns relating to the application of the health shadow price, and the implied (more stringent) cost-effectiveness threshold. In particular, the objectives and the authority of reimbursement bodies, and the feasibility of estimating the health shadow price, may restrict the relevance of the health shadow price threshold when considering the funding of new technologies.

\subsection{The Objective and the Authority of the Reimbursement Body}

Eckermann and Pekarsky start their paper by discussing the possible objective functions relevant to a reimbursement authority such as NICE. They go on to assume that the objective of the reimbursement authority is to achieve allocative efficiency across the entire health system. However, the objectives of NICE appear to be somewhat different, including a responsibility to promote patient access to innovative new medicines and devices, as well as to reduce the perceived postcode lottery in access to treatments more generally.

Primarily, NICE seeks to ensure that the health benefits resulting from newly adopted technologies are not 
outweighed by the health forgone due to their displacement of existing health services. Given a fixed NHS budget, this is equivalent to ensuring that the estimated average ICER of any newly adopted technology is no greater than the ICER of the existing health activity expected to be displaced. This displaced activity is unlikely to be the least cost effective existing activity for a number of reasons, including imperfect information on the part of local decision makers. What matters in determining NICE's threshold is therefore the average ICER of the existing NHS services that are actually displaced in practice.

Where a reimbursement authority has the remit and the capability to control the services from which resources are displaced, and to invest those resources to expand existing services, then the opportunity clearly exists for that authority to adopt an alternative objective than to ensure that more health is expected to be gained than lost from funding new technologies. However, while this may be an option for some reimbursement authorities, it does not appear to be the case for NICE.

Among other responsibilities, NICE is tasked with assessing the clinical and cost effectiveness of new health technologies. Local health authorities have a legal responsibility to follow NICE's technology appraisal recommendations, but NICE cannot issue legally binding guidance on the cost effectiveness of potential reallocations of resources among existing health services [8]. Where NICE issues a recommendation to adopt a new technology that imposes additional costs upon the health system, it is the responsibility of local decision makers to make any necessary disinvestments to accommodate the new technology.

If NICE were to adopt a threshold determined by the health shadow price of the NHS budget, some new technologies with ICERs above this threshold, but below the ICER of displaced services, would be rejected, even though their adoption would be expected to produce more health than they would displace. Indeed, if the most cost effective existing service is less costly and more effective than its comparator, no new technology would be funded unless it too was dominant. This would be contrary to NICE's primary objective. Although Eckermann and Pekarsky are correct to say that NICE's existing approach "does not lead to an increase in the population's health if new technologies are strategically priced at the threshold", they are wrong to say that such a threshold "does not provide an economically meaningful constraint on adopting new technology": the constraint each new technology must meet is that it must be expected to provide at least as much health benefit as the health activity it displaces, which is clearly meaningful given NICE's primary objective.
2.2 The Feasibility of Estimating the Health Shadow Price

There are significant challenges to estimating the health shadow price. In the context of a real-world public healthcare system, it is reasonable to assume that the least and the most cost effective services, and the services that are actually displaced, will vary by geographical area as a result of variation in population needs and in the existing allocation of resources. The health shadow price will therefore be a weighted estimate of the three relevant services across the internal jurisdictions of the health system.

The estimation of the ICER of displaced services is subject to a similar challenge, albeit to a lesser degree. As a result of these difficulties, existing empirical analyses to inform the cost-effectiveness threshold have sought proxy measures. The most recent analysis to inform the UK threshold estimated the ICER of marginal expansions in the healthcare budget over recent years, which reflects the combined effects of spending on new technologies, the expansion of existing services, the displacement of existing services, and increases in the healthcare budget [7].

Although the use of a threshold based upon the ICER of displaced services might not move the health system towards allocative efficiency as swiftly as the use of a threshold based upon the health shadow price, its use nevertheless provides some confidence that the funding of new technologies is not worsening health system performance.

\section{Going Forward}

The health shadow price provides a theoretically sound estimate of the cost-effectiveness threshold from the perspective of moving towards allocative efficiency. However, it is unlikely to be adopted in practice until there are processes in place that actively identify the ICERs of the least cost effective existing activity in contraction and the most cost effective existing activity in expansion, and which facilitate the reallocation of resources between these services.

Eckermann and Pekarsky refer to the theoretical recognition of the health shadow price (opportunity cost) of the resources used to fund new technologies as providing an incentive for publicly funded research on the cost effectiveness of existing technologies. This is explored in more detail in Pekarsky's thesis [9]. The specification of the true health shadow price, and the explicit recognition of the reimbursement of new technologies as a reallocation process of displacement and adoption, is an important step forward in this regard.

If we can start to put values on the potential health gains we are missing due to inefficiencies in both displacement 
and adoption, we can start to move health systems away from institutional processes that focus solely on the funding of new technologies, and towards an integrated process that considers displacement and adoption (of new technologies and expanded or improved existing services) as part of a single process.

The concept of disinvestment lists has been discussed, and some researchers are already compiling lists of lowvalue services and conducting research on processes for managing displacement or disinvestment [10]. A similar process could be envisaged for identifying services with the potential for cost-effective expansion (or cost-effective quality improvement) [11]. This is essentially programme budgeting and marginal analysis on a grand scale, for which extensive stakeholder engagement and political fortitude is required. Resources are also required to generate robust estimates of the incremental costs and effects of services on both lists, and to manage the reallocation process. Based on existing evidence of inefficiencies in all healthcare systems, it is a credible assertion that the payback to the required financial and political investment would justify the substantial investment required.

\section{Discussion}

There is a great deal to commend in Eckermann and Pekarsky's paper. We recommend that readers consult the associated $\mathrm{PhD}$ thesis, which we believe makes a substantial contribution to advancing theory in this area [9].

The use of thresholds based upon Eckermann and Pekarsky's proposals by reimbursement bodies would likely result in fewer new technologies being adopted by public healthcare systems. To the extent that this might provide opportunity for resources to be reallocated into more efficient existing health services, this ought to be welcomed. Nevertheless, the implied consequence that technologies be rejected on the basis that there is a preferred option, but one that cannot be implemented, may be a bridge too far for most reimbursement bodies. This is particularly true for NICE, which has a remit, amongst other things, to support the adoption of innovative new technologies, and which operates in a political environment where the adoption of such a low threshold might be untenable.

In summary, while Eckermann and Pekarsky's approach to determining the cost-effectiveness threshold is theoretically sound, there are a number of issues that must be overcome before it can be of use to reimbursement bodies with a focus upon assessing new health technologies. In the meantime, its practical applicability might be greater among bodies which adopt a specific focus upon considering opportunities for disinvestment and reinvestment among existing healthcare services [12-14].
Acknowledgments Christopher McCabe worked with and for NICE in a number of capacities between 2001 and 2011. He was also a member of the NICE Medical Technologies Advisory Committee. Christopher has provided consultancy services to many pharmaceutical and medical device companies whose revenues might be affected by changes in the NICE cost-effectiveness threshold. Jon Karnon has sat on the economic sub-commitee of the Pharmaceutical Benefits Advisory Committee since 2009. Prior to 2009, he provided consultancy services to a range of pharmaceutical companies whose revenues might be affected by changes in cost-effectiveness thresholds. Mike Paulden declare no conflicts of interest.

\section{References}

1. McCabe C, Claxton K, Culyer AJ. The NICE cost-effectiveness threshold: what it is and what that means. Pharmacoeconomics. 2008;26(9):733-44. http://www.ncbi.nlm.nih.gov/pubmed/18767 894. Accessed 2 Dec 2010

2. Claxton K, Briggs A, Buxton MJ, et al. Value based pricing for NHS drugs: an opportunity not to be missed? BMJ. 2008;336(7638):251-4.

3. Sculpher M, Claxton K. Real economics needs to reflect real decisions: a response to Johnson. Pharmacoeconomics. 2012;30 (2):133-6. doi:10.2165/11596660-000000000-00000.

4. Griffin S, Claxton K, Sculpher M. Decision analysis for resource allocation in health care. J Health Serv Res Policy. 2008;13 (Suppl 3):23-30. doi:10.1258/jhsrp.2008.008017.

5. Culyer A, McCabe C, Briggs A, et al. Searching for a threshold, not setting one: the role of the National Institute for Health and Clinical Excellence. J Health Serv Res Policy. 2007;12(1):56-8. doi:10.1258/135581907779497567.

6. National Institute for Health and Care Excellence. Guide to the methods of technology appraisal 2013. London: NICE; 2013. http://publications.nice.org.uk/pmg9. Accessed 28 Feb 2014.

7. Claxton K, Martin S, Soares M, Rice N, Spackman E, Hinde S, et al. Methods for the estimation of the NICE cost effectiveness threshold final report, University of York. 2013. http://www.york. ac.uk/media/che/documents/papers/researchpapers/CHERP81_ methods_estimation_NICE_costeffectiveness_threshold_\%28Nov 2013\%29.pdf. Accessed 28 Feb 2014.

8. Garner S, Littlejohns P. Disinvestment from low value clinical interventions: NICEly done? BMJ. 2011;343:d4519. doi:10.1136/ bmj.d4519.

9. Pekarsky B. Trust, constraints and the counterfactual: Reframing the political economy of new drugs. 2012;(April) [thesis]. http:// digital.library.adelaide.edu.au/dspace/bitstream/2440/79171/3/02 whole.pdf. Accessed 28 Feb 2014.

10. Elshaug AG, Watt AM, Mundy L, Willis CD. Over 150 potentially low-value health care practices: an Australian study. Med J Aust. 2012;197(10):556-60. doi:10.5694/mja12.11083.

11. Karnon J, Caffrey O, Pham C, et al. Applying risk adjusted costeffectiveness (RAC-E) analysis to hospitals: estimating the costs and consequences of variation in clinical practice. Health Econ. 2013;22(6):631-42. doi:10.1002/hec.2828.

12. ABIM Foundation. Choosing wisely. 2014. http://www. choosingwisely.org/. Accessed 12 Feb 2014.

13. Institute for Healthcare Improvement. The IHI Triple Aim. 2013. http://www.ihi.org/engage/initiatives/tripleaim/pages/default.aspx. Accessed 28 Feb 2014.

14. Porter ME. What is value in health care? N Engl J Med. 2010;363(26):2477-81. doi:10.1056/NEJMp1011024.

15. Eckermann S, Pekarsky B. Can the real opportunity cost stand up: displaced services, the straw man outside the room. Pharmacoeconomics. Epub 2014 Feb. doi:10.1007/s40273-014-0140-3. 